\title{
WEAKLY PROTECTED NODES IN RANDOM BINARY SEARCH TREES
}

\author{
Ezzat Mohammad Nezhad ${ }^{1}$, Mehri Javanian ${ }^{2, *}$ \\ AND RAMIN IMANY NABIYYI ${ }^{1}$
}

\begin{abstract}
Here, we derive the exact mean and variance of the number of weakly protected nodes (the nodes that are not leaves and at least one of their children is not a leaf) in binary search trees grown from random permutations. Furthermore, by using contraction method, we prove normal limit law for a properly normalized version of this tree parameter.
\end{abstract}

Mathematics Subject Classification. 05C05, 05C80, $60 \mathrm{~F} 05$.

Received May 23, 2021. Accepted January 17, 2022.

\section{DEFinitions}

Let $P=\left(p_{1}, p_{2}, \ldots, p_{n}\right)$ be a uniformly random permutation of $\{1,2, \ldots, n\}$. A random binary search tree is generated by $P$ as follows. The elements of $P$ serve as keys. The keys are stored in the internal nodes of the tree. The root of the tree stores the first key $p_{1}$. The second key $p_{2}$ is compared with $p_{1}$. If $p_{2}<p_{1}$, then $p_{2}$ becomes root of the left subtree; otherwise, $p_{2}$ becomes root of the right subtree. The process repeats on subsequent keys in the same manner. Note that a uniform probability distribution on permutations does not induce a uniform probability distribution on binary search trees [5]. Figure 1 shows an example of a binary search tree.

In a rooted tree, a protected node is a node that is not a leaf and none of its children is a leaf. For many types of random trees, protected nodes have been investigated in numerous papers, see for instance $[1-4,6]$.

By a weakly protected node, we mean a node that is not a leaf and at least one of its children is not a leaf. Figure 1 illustrates the protected nodes and weakly protected nodes in a binary search tree.

In this note, we study the number of weakly protected nodes in random binary search trees. Recently, the number of weakly protected nodes have only been studied for ordered trees in [10].

\section{THE EXPECTATION AND VARIANCE}

We denote the number of weakly protected nodes in a random binary search tree of size $n$ by $X_{n}$. We denote the sizes of the left subtree and right subtree of the root by $U_{n}$ and $n-1-U_{n}$, respectively. In view of the probability distribution on binary search trees, $U_{n}$ and $n-1-U_{n}$ have uniform distribution on the set $\{0,1, \ldots, n-1\}$.

Keywords and phrases: Random binary search tree, Weakly protected nodes, Zolotarev metric, Contraction method, limiting distribution.

1 Department of Statistics, Faculty of Mathematical Sciences, University of Tabriz, Tabriz Iran.

2 Department of Statistics, Faculty of Sciences, University of Zanjan, Zanjan, Iran.

* Corresponding author: javanian@znu.ac.ir 


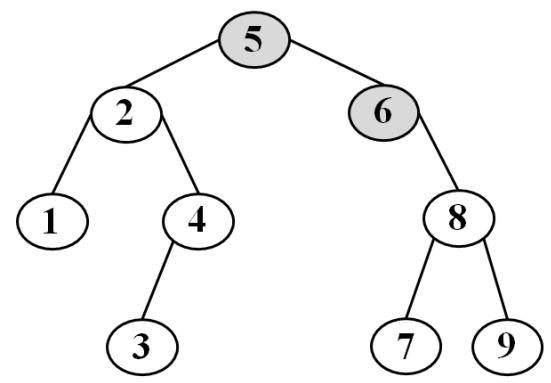

(a)

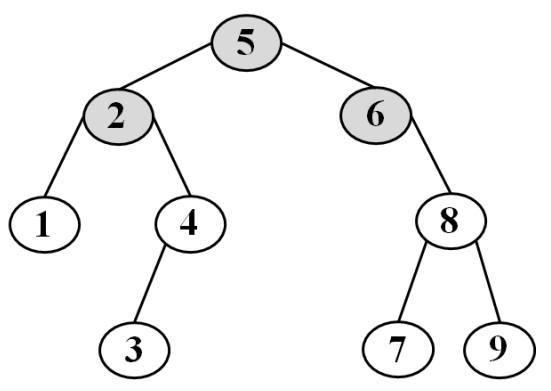

(b)

FiguRE 1. A binary search tree built from the keys $(5,2,6,1,8,7,4,9,3)$ where gray nodes are: (a) Protected nodes; (b) Weakly protected nodes.

Let the notation $\stackrel{d}{=}$ indicate the equality in distribution. For $n \geq 3$, we have a distributional recurrence for $X_{n}$, i.e,

$$
X_{n} \stackrel{d}{=} X_{U_{n}}+\bar{X}_{n-1-U_{n}}+1-\delta_{n, 3} \mathbb{1}_{\left\{U_{n}=1\right\}},
$$

where $\mathbb{1}_{A}$ is the indicator function of $A, \delta_{i, j}$ is Kronecker delta, $X_{n} \stackrel{d}{=} \bar{X}_{n}$, and $X_{n}, \bar{X}_{n}$ and $U_{n}$ are independent. Moreover, $X_{n}=0$ for $n=0,1,2$.

Theorem 2.1. Let $X_{n}$ denote the number of weakly protected nodes in a random binary search tree of size $n$. Then

$$
\begin{aligned}
\mathbb{E}\left(X_{n}\right) & =\frac{7 n-8}{15}, \quad \text { for } n \geq 4, \\
\operatorname{Var}\left(X_{n}\right) & =\frac{211}{3150}(n+1), \quad \text { for } n \geq 10,
\end{aligned}
$$

with $\mathbb{E}\left(X_{n}\right)=0$ for $n=0,1,2$ and $\mathbb{E}\left(X_{3}\right)=\frac{2}{3}$. Moreover,

$$
\begin{array}{llll}
\operatorname{Var}\left(X_{n}\right)=0, & n=0,1,2, & \operatorname{Var}\left(X_{3}\right)=\frac{2}{9}, \\
\operatorname{Var}\left(X_{4}\right)=\frac{2}{9}, & \operatorname{Var}\left(X_{5}\right)=\frac{32}{75}, & \operatorname{Var}\left(X_{6}\right)=\frac{38}{75}, \\
\operatorname{Var}\left(X_{7}\right)=\frac{286}{525}, & \operatorname{Var}\left(X_{8}\right)=\frac{211}{350}, & \operatorname{Var}\left(X_{9}\right)=\frac{211}{315} .
\end{array}
$$

Proof. Taking expectation of (2.1), for $n \geq 3$, we obtain

$$
\mathbb{E}\left(X_{n}\right)=\mathbb{E}\left(X_{U_{n}}\right)+\mathbb{E}\left(\bar{X}_{n-1-U_{n}}\right)+1-\frac{\delta_{n, 3}}{n} .
$$

By conditioning on $U_{n}$, the equation (2.4) gives

$$
n \mathbb{E}\left(X_{n}\right)=2 \sum_{j=0}^{n-1} \mathbb{E}\left(X_{j}\right)+n-\delta_{n, 3} .
$$


We subtract from the equation (2.5) a version of itself with $n$ replaced by $n-1$ and unwind the recurrence: for $n \geq 4$,

$$
\begin{aligned}
\mathbb{E}\left(X_{n}\right) & =\frac{n+1}{n} \mathbb{E}\left(X_{n-1}\right)+\frac{1+\delta_{n-1,3}}{n} \\
& \vdots \\
& =\frac{n+1}{4} \mathbb{E}\left(X_{3}\right)+\sum_{j=3}^{n-1} \frac{(n+1)\left(1+\delta_{j, 3}\right)}{(j+1)(j+2)} \\
& =\frac{4(n+1)}{15}-\frac{1}{n+2}+\sum_{j=5}^{n+1} \frac{n+1}{j}-\sum_{j=5}^{n+1} \frac{n+1}{j+1} .
\end{aligned}
$$

By simplifying this we get (2.2). Similarly, by (2.1) and (2.2), we have, for $n \geq 9$,

$$
\begin{aligned}
\mathbb{E}\left(X_{n}^{2}\right) & =\frac{2}{n} \sum_{j=0}^{n-1} \mathbb{E}\left(X_{j}^{2}\right)+\frac{4}{n} \sum_{j=4}^{n-1} \mathbb{E}\left(X_{j}\right)+\frac{4}{n} \mathbb{E}\left(X_{3}\right)+1 \\
& +\frac{2}{n} \sum_{j=4}^{n-5} \mathbb{E}\left(X_{j}\right) \mathbb{E}\left(X_{n-1-j}\right)+\frac{4}{n} \mathbb{E}\left(X_{3}\right) \mathbb{E}\left(X_{n-4}\right) \\
& =\frac{2}{n} \sum_{j=0}^{n-1} \mathbb{E}\left(X_{j}^{2}\right)+\frac{4}{n} \sum_{j=4}^{n-1} \frac{7 j-8}{15}+\frac{8}{3 n}+1 \\
& +\frac{2}{n} \sum_{j=4}^{n-5} \frac{7 j-8}{15} \cdot \frac{7(n-1-j)-8}{15}+\frac{8}{3 n} \cdot \frac{7(n-4)-8}{15} \\
& =\frac{2}{n} \sum_{j=0}^{n-1} \mathbb{E}\left(X_{j}^{2}\right)+\frac{49}{675} n^{2}+\frac{49}{225} n-\frac{577}{675}+\frac{112}{45 n} .
\end{aligned}
$$

By the last equation, for $n \geq 10$, it follows that

$$
\begin{aligned}
n \mathbb{E}\left(X_{n}^{2}\right) & -(n-1) \mathbb{E}\left(X_{n-1}^{2}\right) \\
& =2 \mathbb{E}\left(X_{n-1}^{2}\right)+\frac{49}{225} n^{2}+\frac{49}{225} n-1 .
\end{aligned}
$$

Therefore, for $n \geq 10$, we obtain

$$
\begin{aligned}
\mathbb{E}\left(X_{n}^{2}\right) & =\frac{n+1}{n} \mathbb{E}\left(X_{n-1}^{2}\right)+\frac{1}{n}\left(\frac{49}{225} n^{2}+\frac{49}{225} n-1\right) \\
& \vdots \\
& =\frac{n+1}{10} \mathbb{E}\left(X_{9}^{2}\right)+\sum_{j=10}^{n} \frac{n+1}{j(j+1)}\left(\frac{49}{225} j^{2}+\frac{49}{225} j-1\right) \\
& =\frac{49}{225} n^{2}-\frac{1357}{3150} n+\frac{123}{350}
\end{aligned}
$$

with $\mathbb{E}\left(X_{3}^{2}\right)=\frac{2}{3}, \mathbb{E}\left(X_{4}^{2}\right)=2, \mathbb{E}\left(X_{5}^{2}\right)=\frac{11}{3}, \mathbb{E}\left(X_{6}^{2}\right)=\frac{254}{45}, \mathbb{E}\left(X_{7}^{2}\right)=\frac{505}{63}, \mathbb{E}\left(X_{8}^{2}\right)=\frac{759}{70}$ and $\mathbb{E}\left(X_{9}^{2}\right)=\frac{494}{35}$. 
Finally, applying (2.6) and $\operatorname{Var}\left(X_{n}\right)=\mathbb{E}\left(X_{n}^{2}\right)-\left(\mathbb{E}\left(X_{n}\right)\right)^{2}$, the assertion in (2.3) follows.

\section{LIMITING DISTRIBUTION}

In this section, we begin to prove the normality of limiting distribution of $X_{n}$. The proof was completed by applying the contraction method, which was first introduced by [9], in studying the Quicksort algorithm.

Here, we prefer the Zolotarev metric $\zeta_{3}$ (see [8]) as the metric space applied in the contraction method. Let the distribution of a random variable $X$ denoted by $\mathcal{L}(X)$. Then, for any given random variables $X$ and $Y$, the 3rd order Zolotarev metric between $X$ and $Y$ is defined as

$$
\begin{aligned}
\zeta_{3}(X, Y) & =\zeta_{3}(\mathcal{L}(X), \mathcal{L}(Y)) \\
& :=\sup \{|\mathbb{E}[f(X)]-\mathbb{E}[f(Y)]|: f \in \mathcal{F}\}
\end{aligned}
$$

where $\mathcal{F}=\left\{f: f \in C^{(2)},\left|f^{(2)}(x)-f^{(2)}(y)\right| \leq|x-y|\right\}$ denotes the set of all twice differentiable functions, where the second derivative is Lipschitz continuous with Lipschitz constant equal to 1.

The following lemma gives several properties of probability metric $\zeta_{3}(X, Y)$, which are quite useful in our proof of Theorem 3.5.

Lemma 3.1 (see [8]). Let $\zeta_{3}(X, Y)$ be the 3rd order Zolotarev metric between the random variables $X$ and $Y$. Then,

(i) For any real number $c>0$,

$$
\zeta_{3}(c X, c Y)=c^{3} \zeta_{3}(X, Y)
$$

(ii) If the random variables $Y$ and $\left(X_{1}, X_{2}\right)$ are independent mutually, then

$$
\zeta_{3}\left(X_{1}+Y, X_{2}+Y\right) \leq \zeta_{3}\left(X_{1}, X_{2}\right)
$$

(iii) For any random variables $X$ and $Y$,

$$
\begin{aligned}
\mathbb{E}\left(|X|^{3}\right)+\mathbb{E}\left(|Y|^{3}\right) & <\infty, \quad \mathbb{E}\left(X^{k}\right)=\mathbb{E}\left(Y^{k}\right), k=1,2 \\
& \Longleftrightarrow \zeta_{3}(X, Y)<\infty
\end{aligned}
$$

(iv) For the random variables $V$ and $\left\{V_{n}\right\}_{n \geq 1}$, as $n \rightarrow \infty$

$$
\zeta_{3}\left(V_{n}, V\right) \rightarrow 0 \Longrightarrow V_{n} \stackrel{\mathcal{D}}{\longrightarrow} V
$$

where the notation $\stackrel{\mathcal{D}}{\longrightarrow}$ denotes the convergence in distribution.

Lemma 3.2 (see [7]). let $X_{1}, X_{2}, T_{1}$ and $T_{2}$ be random variables such that the pairs $\left(X_{1}+T_{1}, X_{2}+T_{2}\right)$ and $\left(X_{1}, X_{2}\right)$ satisfies (3.3). Then

$$
\begin{aligned}
& \zeta_{3}\left(X_{1}+T_{1}, X_{2}+T_{2}\right) \leq \zeta_{3}\left(X_{1}, X_{2}\right) \\
& +\sum_{i=1}^{2}\left\{\frac{\left\|X_{i}\right\|_{3}^{2}\left\|T_{i}\right\|_{3}}{2}+\frac{\left\|X_{i}\right\|_{3}\left\|T_{i}\right\|_{3}^{2}}{2}+\frac{\left\|T_{i}\right\|_{3}^{3}}{6}\right\},
\end{aligned}
$$

where $\|X\|_{3}:=\mathbb{E}\left(|X|^{3}\right)^{1 / 3}$ for a random variable $X$. 
Moreover, the proof of Theorem 3.5 requires the following upper bound for metric $\zeta_{3}$ :

$$
\zeta_{3}(X, Y) \leq \frac{1}{2}\left(\|X\|_{3}^{2}+\|X\|_{3}\|Y\|_{3}+\|Y\|_{3}^{2}\right) \ell_{3}(X, Y)
$$

where the minimal $L_{3}$-metric $\ell_{3}$ defined by

$$
\begin{aligned}
\ell_{3}(X, Y) & :=\ell_{3}(\mathcal{L}(X), \mathcal{L}(Y)) \\
& :=\inf \left\{\left\|X^{\prime}-Y^{\prime}\right\|_{3}: X \stackrel{d}{=} X^{\prime}, Y \stackrel{d}{=} Y^{\prime}\right\}
\end{aligned}
$$

for random variables $X$ and $Y$ with $\|X\|_{3}<\infty,\|Y\|_{3}<\infty$.

We standardize $X_{n}$ with its mean and variance, i.e.,

$$
Y_{n}:=\frac{X_{n}-\mathbb{E}\left(X_{n}\right)}{\sigma(n)}, \quad \sigma^{2}(n):=\operatorname{Var}\left(X_{n}\right)
$$

Let denote a quantity

$$
Y_{n} \stackrel{d}{=} \frac{\sigma\left(U_{n}\right)}{\sigma(n)} Y_{U_{n}}+\frac{\sigma\left(n-1-U_{n}\right)}{\sigma(n)} \bar{Y}_{n-1-U_{n}}, \quad n \geq 4
$$

where $Y_{i} \stackrel{d}{=} \bar{Y}_{i}$, for $0 \leq i \leq n-1$. The random variables $Y_{i}, \bar{Y}_{i}, U_{n}, 0 \leq i \leq n-1$, are independent. To prove the Theorem 3.5, we still require some more arrangements. The following three lemmas are necessary.

Lemma 3.3. Let $W, W_{1}$ and $W_{2}$ be independent standard normal random variables. Then we have

$$
W \stackrel{d}{=} \sqrt{\frac{U_{n}+1}{n+1}} W_{1}+\sqrt{\frac{n-U_{n}}{n+1}} W_{2},
$$

where $U_{n}$ is a random variable with uniform distribution on the set $\{0,1, \ldots, n-1\}$.

Proof. It is sufficient to verify that the characteristic function of the right side of (3.6) is the same as that of a standard normal random variable. From the independence of the random variables $W, W_{1}, W_{2}, U_{n}$, we have

$$
\begin{aligned}
& \mathbb{E}\left[\exp \left\{i t\left(\sqrt{\frac{U_{n}+1}{n+1}} W_{1}+\sqrt{\frac{n-U_{n}}{n+1}} W_{2}\right)\right\}\right] \\
= & \sum_{j=0}^{n-1} \frac{1}{n} \mathbb{E}\left[\exp \left\{i t\left(\sqrt{\frac{j+1}{n+1}} W_{1}+\sqrt{\frac{n-j}{n+1}} W_{2}\right)\right\}\right] \\
= & \sum_{j=0}^{n-1} \frac{1}{n} \mathbb{E}\left[\exp \left\{i t W_{1} \sqrt{\frac{j+1}{n+1}}\right\}\right] \mathbb{E}\left[\exp \left\{i t W_{2} \sqrt{\frac{n-j}{n+1}}\right\}\right] \\
= & \sum_{j=0}^{n-1} \frac{1}{n} \exp \left(-\frac{j+1}{n+1} \cdot \frac{t^{2}}{2}\right) \exp \left(-\frac{n-j}{n+1} \cdot \frac{t^{2}}{2}\right)=e^{-\frac{t^{2}}{2}}
\end{aligned}
$$

where, the function $e^{-\frac{t^{2}}{2}}$ is the characteristic function of a standard normal random variable. Hence we obtain the claim.

Lemma 3.4. As $n \rightarrow \infty, \mathbb{E}\left[\left|Y_{n}^{3}\right|\right]=\mathcal{O}(1)$. 
Proof. By Lyapunov's inequality, $\mathbb{E}\left[\left|Y_{n}\right|\right] \leq \sqrt{\mathbb{E}\left[\left|Y_{n}\right|^{2}\right]}=1$. Let $\xi_{n}:=1 \vee \max _{0 \leq j \leq n} \mathbb{E}\left[\left|Y_{j}^{3}\right|\right]$ and $U$ be a uniform random variable on $(0,1)$. Then from $(2.1)$ we obtain

$$
\begin{aligned}
\mathbb{E}\left[\left|Y_{n}^{3}\right|\right] & \leq 2 \sum_{j=0}^{n-1} \frac{1}{n}\left(\frac{\sigma(j)}{\sigma(n)}\right)^{3} \mathbb{E}\left[\left|Y_{j}\right|^{3}\right]+\mathcal{O}(1) \\
& \leq\left(2 \mathbb{E}\left(U^{\frac{3}{2}}\right)+o(1)\right) \xi_{n-1}+\mathcal{O}(1) \\
& \leq(0.8+o(1)) \xi_{n-1}+\mathcal{O}(1) .
\end{aligned}
$$

Hence, there exist an $n_{0} \in \mathbb{N}$ and a constant $0<\alpha<\infty$ such that for $n \geq n_{0}$

$$
\mathbb{E}\left[\left|Y_{n}^{3}\right|\right] \leq 0.9 \xi_{n-1}+\alpha .
$$

By induction, we have $\mathbb{E}\left[\left|Y_{n}^{3}\right|\right] \leq \xi_{n_{0}} \vee(10 \alpha)$ for all $n \geq 0$. This implies the claim.

In the following, we begin to prove the asymptotic normality distribution for $X_{n}$.

Theorem 3.5. Let $X_{n}$ denote the number of weakly protected nodes in a random binary search tree of size $n$. Then,

$$
\frac{X_{n}-\frac{7}{15} n}{\sqrt{n}} \stackrel{\mathcal{D}}{\longrightarrow} \mathcal{N}\left(0, \frac{211}{3150}\right), \quad \text { as } n \rightarrow \infty,
$$

where $\mathcal{N}\left(\mu, \sigma^{2}\right)$ denotes a normal random variable with mean $\mu$ and variance $\sigma^{2}$.

Proof. By (3.4), we just need to show that the Zolotarev metric between the random variables $Y_{n}$ and $N$, a standard normal random variable, approaches 0 , as $n \rightarrow \infty$. For $W_{1}$ and $W_{2}$ independent standard normal random variables, also independent of $U_{n}$, we set

$$
\Theta_{n}:=\frac{\sigma\left(U_{n}\right)}{\sigma(n)} W_{1}+\frac{\sigma\left(n-1-U_{n}\right)}{\sigma(n)} W_{2}, \quad n \geq 4
$$

Note that $\operatorname{Var}\left(\Theta_{n}\right)>0$ for all $n \geq n_{0}$, and $\operatorname{Var}\left(\Theta_{n}\right) \rightarrow 1$ as $n \rightarrow \infty$. Hence there exists a deterministic sequence $\left(\delta_{n}\right)_{n \geq n_{0}}$ with $\delta_{n} \rightarrow 0$ as $n \rightarrow \infty$ such that $\operatorname{Var}\left(\left(1+\delta_{n}\right) \Theta_{n}\right)=1$ for all $n \geq 4$. So, by Lemma 3.4, each pair from the random variables $Y_{n},\left(1+\delta_{n}\right) \Theta_{n}$ and $N$ satisfies (3.3). Thus we obtain

$$
\zeta_{3}\left(Y_{n}, N\right) \leq \zeta_{3}\left(Y_{n},\left(1+\delta_{n}\right) \Theta_{n}\right)+\zeta_{3}\left(\left(1+\delta_{n}\right) \Theta_{n}, N\right) .
$$

Now Lemma 3.2 yields

$$
\zeta_{3}\left(Y_{n},\left(1+\delta_{n}\right) \Theta_{n}\right) \leq \zeta_{3}\left(Y_{n}, \Theta_{n}\right)+o(1)
$$

Using the bound (3.5) and Lemma 3.3, for some finite constant $M>0$, we obtain

$$
\begin{aligned}
\zeta_{3}\left(\left(1+\delta_{n}\right) \Theta_{n}, N\right) \leq M \ell_{3}\left(\left(1+\delta_{n}\right) \Theta_{n}, N\right) \\
\quad \leq M \|\left(\left(1+\delta_{n}\right) \frac{\sigma\left(U_{n}\right)}{\sigma(n)}-\sqrt{\frac{U_{n}+1}{n+1}}\right) W_{1} \\
\quad+\left(\left(1+\delta_{n}\right) \frac{\sigma\left(n-1-U_{n}\right)}{\sigma(n)}-\sqrt{\frac{n-U_{n}}{n}}\right) W_{2} \|_{3} \rightarrow 0 .
\end{aligned}
$$


From (3.1) and (3.2), we can conclude that

$$
\begin{aligned}
\zeta_{3}\left(Y_{n}, N\right) \leq & \left(Y_{n}, \Theta_{n}\right)+o(1) \\
\leq & \zeta_{3}\left(\frac{\sigma\left(U_{n}\right)}{\sigma(n)} Y_{U_{n}}+\frac{\sigma\left(n-1-U_{n}\right)}{\sigma(n)} \bar{Y}_{n-1-U_{n}},\right. \\
& \left.\frac{\sigma\left(U_{n}\right)}{\sigma(n)} W_{1}+\frac{\sigma\left(n-1-U_{n}\right)}{\sigma(n)} W_{2}\right)+o(1) \\
\leq & \sum_{j=0}^{n-1} \frac{1}{n} \zeta_{3}\left(\frac{\sigma(j)}{\sigma(n)} Y_{j}+\frac{\sigma(n-1-j)}{\sigma(n)} \bar{Y}_{n-1-j},\right. \\
& \left.\frac{\sigma(j)}{\sigma(n)} W_{1}+\frac{\sigma(n-1-j)}{\sigma(n)} W_{2}\right)+o(1) \\
= & 2 \sum_{j=0}^{n-1} \frac{1}{n}\left(\frac{\sigma(j)}{\sigma(n)}\right)^{3} \zeta_{3}\left(Y_{j}, N\right)+o(1) \\
= & 2 \mathbb{E}\left[\left(\frac{\sigma\left(U_{n}\right)}{\sigma(n)}\right)^{3} \zeta_{3}\left(Y_{U_{n}}, N\right)\right]+o(1) \\
\leq & \left(2 \mathbb{E}\left(U^{\frac{3}{2}}\right)+o(1)\right)_{0 \leq j \leq n-1} \zeta_{3}\left(Y_{j}, N\right)+o(1) .
\end{aligned}
$$

This implies, similarly to the inequality $(3.7),\left(\zeta_{3}\left(Y_{n}, N\right)\right)_{n \geq 0}$ that is bounded. We denote $\xi:=\sup _{n \geq 0} \zeta_{3}\left(Y_{n}, N\right)$ and $s:=\limsup _{n \rightarrow \infty} \zeta_{3}\left(Y_{n}, N\right) \geq 0$. For any $\varepsilon>0$ there exists an $n_{1} \geq 4$ such that $\zeta_{3}\left(Y_{n}, N\right) \leq s+\varepsilon$ for all $n \geq n_{1}$. Hence, from (3.8) we obtain

$$
\begin{aligned}
\zeta_{3}\left(Y_{n}, N\right) & \leq 2 \mathbb{E}\left[\mathbb{1}_{\left\{U_{n} \leq n_{1}\right\}}\left(\frac{\sigma\left(U_{n}\right)}{\sigma(n)}\right)^{3}\right] \xi \\
& +2 \mathbb{E}\left[\mathbb{1}_{\left\{U_{n}>n_{1}\right\}}\left(\frac{\sigma\left(U_{n}\right)}{\sigma(n)}\right)^{3}\right](s+\varepsilon)+o(1) \\
& \sim 2 \mathbb{E}\left[\mathbb{1}_{\left\{U_{n}>n_{1}\right\}}\left(\frac{\sigma\left(U_{n}\right)}{\sigma(n)}\right)^{3}\right](s+\varepsilon)+o(1) .
\end{aligned}
$$

So $0 \leq s=\limsup _{n \rightarrow \infty} \zeta_{3}\left(Y_{n}, N\right) \leq 0.8(s+\varepsilon)<s+\varepsilon$. Since $\varepsilon>0$ is arbitrary then we have $s=0$. Therefore, by (3.4), the assertion holds.

\section{REFERENCES}

[1] M. Bona, $k$-protected nodes in binary search trees. Adv. Appl. Math. 53 (2014) 1-11.

[2] L. Devroye and S. Janson, Protected nodes and fringe subtrees in some random trees. Electr. Commun. Probab. 19 (2014) 1-10.

[3] C. Holmgren and S. Janson, Asymptotic distribution of two-protected nodes in ternary search trees. Electr. J. Probab. 20 (2015) 1-20.

[4] C. Holmgren and S. Janson, Limit laws for functions of fringe trees for binary search trees and recursive trees. Electr. J. Probab. 20 (2015) 1-51.

[5] H.M. Mahmoud, Evolution of random search trees. John Wiley \& Sons Inc., New York (1992).

[6] H.M. Mahmoud and M.D. Ward, Asymptotic distribution of two-protected nodes in random binary search trees. Appl. Math. Lett. 25 (2012) 2218-2222.

[7] R. Neininger, Refined quicksort asymptotics. Random Struct. Algor. 46 (2015) 346-361.

[8] S. Rachev, Probability Metrics and the Stability of Stochastic Models. New York (1991). 
[9] U. Roesler, A limit theorem for "Quicksort". RAIRO: ITA 25 (1991) 85-100.

[10] L. Yang and S.L. Yang, Weakly protected points in ordered trees. Graphs Combinat. (2021) https://doi.org/10.1007/ s00373-021-02278-w.

\section{Subscribe to Open (S2O) A fair and sustainable open access model}

This journal is currently published in open access under a Subscribe-to-Open model (S2O). S2O is a transformative model that aims to move subscription journals to open access. Open access is the free, immediate, online availability of research articles combined with the rights to use these articles fully in the digital environment. We are thankful to our subscribers and sponsors for making it possible to publish this journal in open access, free of charge for authors.

Please help to maintain this journal in open access!

Check that your library subscribes to the journal, or make a personal donation to the $\mathrm{S} 2 \mathrm{O}$ programme, by contacting subscribers@edpsciences.org

More information, including a list of sponsors and a financial transparency report, available at: https://www.edpsciences.org/en/maths-s2o-programme 